\title{
Organotypic specificity of key RET adaptor-docking sites in the pathogenesis of neurocristopathies and renal malformations in mice
}

\author{
Sanjay Jain,, ${ }^{1,3}$ Amanda Knoten, ${ }^{1}$ Masato Hoshi, ${ }^{1}$ Hongtao Wang, ${ }^{4}$ Bhupinder Vohra, ${ }^{5}$ \\ Robert O. Heuckeroth, ${ }^{3,4,6}$ and Jeffrey Milbrandt'1,2,3,5
}

\begin{abstract}
${ }^{1}$ Department of Internal Medicine (Renal Division), ${ }^{2}$ Department of Pathology, ${ }^{3} \mathrm{HOPE}$ Center for Neurological Disorders, ${ }^{4}$ Department of Pediatrics, ${ }^{5}$ Department of Genetics, and ${ }^{6}$ Department of Developmental Biology, Washington University School of Medicine, St. Louis, Missouri.
\end{abstract}

\begin{abstract}
The receptor tyrosine kinase ret protooncogene (RET) is implicated in the pathogenesis of several diseases and in several developmental defects, particularly those in neural crest-derived structures and the genitourinary system. In order to further elucidate RET-mediated mechanisms that contribute to these diseases and decipher the basis for specificity in the pleiotropic effects of RET, we characterized development of the enteric and autonomic nervous systems in mice expressing RET9 or RET51 isoforms harboring mutations in tyrosine residues that act as docking sites for the adaptors Plc $\gamma$, Src, Shc, and Grb2. Using this approach, we found that development of the genitourinary system and the enteric and autonomic nervous systems is dependent on distinct RET-stimulated signaling pathways. Thus, mutation of RET51 at Y1062, a docking site for multiple adaptor proteins including Shc, caused distal colon aganglionosis reminiscent of Hirschsprung disease (HSCR). On the other hand, this mutation in RET9, which encodes an isoform that lacks the Grb2 docking site present in RET51, produced severe abnormalities in multiple organs. Mutations that abrogate RET-Plcy binding, previously shown to produce features reminiscent of congenital anomalies of kidneys or urinary tract (CAKUT) syndrome, produced only minor abnormalities in the nervous system. Abrogating RET51-Src binding produced no major defects in these systems. These studies provide insight into the basis of organotypic specificity and redundancy in RET signaling within these unique systems and in diseases such as HSCR and CAKUT.
\end{abstract}

\section{Introduction}

Aberrant signaling by receptor tyrosine kinases (RTKs) leads to congenital malformations, cancer, and stem cell renewal defects. Upon activation, RTKs typically interact with intracellular adaptor proteins (PLC $\gamma$, SRC, SHC, GRB2) via docking phosphotyrosines to activate intracellular signaling cascades, such as PKC, AKT, and MAPK, that regulate cellular proliferation, survival, migration, and self renewal (1). While studies of mutant mice have elucidated physiological roles of several RTKs, little is known about the relative contributions of individual signaling cascades in producing the diverse phenotypes that result from RTK dysfunction.

Mutations in rearranged during transfection (RET) protooncogene, a transmembrane RTK, cause multiple human diseases, particularly those affecting neural crest-derived structures (enteric ganglia, parasympathetic and sympathetic ganglia, thyroid C cells, and adrenal medulla) (2). Inactivating RET mutations are a common cause of Hirschsprung disease (HSCR) (3), or distal intestinal aganglionosis, as well as renal agenesis (4). In contrast, activating RET mutations lead to human multiple endocrine neoplasia (MEN) syndromes 2A and 2B, 2 life-threatening familial cancer predisposition syndromes. Recently, RET mutations have been found in patients presenting with multiple abnormalities, including HSCR, with congenital anomalies of kidneys or urinary tract

Conflict of interest: J. Milbrandt and Washington University may derive benefit from a licensing agreement with Ceregene, which did not provide any support for this work. Citation for this article: J Clin Invest. 2010;120(3):778-790. doi:10.1172/JCI41619.
(CAKUT), or HSCR and MEN2, or CAKUT and MEN2 (5-7). It is unclear how RET-mediated signaling cascades lead to anomalies in isolated or combined presentation of these anomalies.

RET signaling is activated by the formation of a receptor complex that includes a member of the glial cell line-derived neurotrophic factor (GDNF) family ligands (GFLs), which includes GDNF, Neurturin, Artemin, and Persephin, along with a GDNF receptor $\alpha(G F R \alpha)$ coreceptor (GFR $\alpha 1-4)(8)$. The specificity of these interactions is dependent on the coreceptor-ligand interaction, with the RET tyrosine kinase serving as a common signaling component. A number of studies in mice have defined the importance of Ret signaling in the development and maintenance of the peripheral nervous system (PNS), with particular emphasis on the autonomic nervous system (ANS), enteric nervous system (ENS), and the urinary and reproductive systems (9-13). For example, mice lacking Gdnf, its cognate coreceptor Gfr $\alpha 1$, or Ret all have similar and severe deficits that include complete intestinal aganglionosis, agenesis of sphenopalatine ganglia, migration and neuronal projection defects in the sympathetic ganglia, and bilateral renal agenesis $(10,14-20)$.

RET activation results in phosphorylation of key docking tyrosines that bind to several intracellular adaptor proteins such as SRC (at Y981), PLC $\gamma$ (at Y1015), SHC, FRS2, IRS1 and -2, ENIGMA, DOKs 4, 5 and 6 (at Y1062), and GRB2 (at Y1096) (21). Recruitment of SRC to RET Y981 activates the MAPK pathway, while recruitment of PLC $\gamma$ binding to RET Y1015 primarily activates the PKC pathway (21). The RET Y1062 phosphotyrosine serves as a dock- 
Table 1

List of RET-mutant mice used

Homozygous mutant studied
Ret $^{\text {RETg/RET9 }}$
Ret $^{\text {RET51/RET51 }}$
Ret $^{\text {RET51(Y981F)/RET51(Y981F) }}$
$\operatorname{Ret}^{\text {RET51(Y1015F)/RET51(Y1015F) }}$
$\operatorname{Ret}^{\text {RET51(Y1062F)/RET51(Y1062F) }}$
$\operatorname{Ret}^{\text {RET51(Cdel)/RET51(Cdel) }}$
Ret $^{\text {RET9(Y981F)/RET9(Y981F) }}$
Ret $^{\text {RET9(Y1015F)/RET9(Y1015F) }}$
$\operatorname{Ret}^{\text {RET9(Y1062F)/RET9(Y1062F) }}$
$\operatorname{Ret}^{\text {RET9(51C)/RET9(51C) }}$

Shown are the homozygous mutant mice expressing the knocked in human RET alleles and the simplified allele names that are used in the manuscript.

ing site for multiple adaptors. It primarily supports SHC binding to recruit either the GRB2-SOS complex leading to MAPK activation or GRB2-GAB1 to stimulate PI3K/AKT signaling. GRB2 also binds directly to phospho-Y1096, where it preferentially activates PI3K/AKT signaling (22).

There are 2 major alternative splice isoforms of RET, RET9 and RET51, that may differentially modulate RET-activated intracellular signaling cascades. RET9 (1072 aa) and RET51 (1114 aa) diverge after residue 1063 and thus are identical except for the C-terminal cytoplasmic domain (23). RET51 carboxyl terminus contains an additional docking tyrosine, Y1096, for GRB2. The physiologic roles of these 2 isoforms and the signaling pathways emanating from specific phosphorylated tyrosine docking sites have been studied in vitro, but information in vivo is limited. The relative importance of the RET isoforms and particular docking sites has differed depending on the system investigated (24-27). For example, in mice exclusively expressing chimeric mouse-human WT Ret 9 or Ret51, only Ret 9 supported normal kidney or ENS development (28). However, normal kidney development is supported in mice expressing either WT human RET9 or RET51. Only when individual RET docking sites were mutated did the distinct roles of these key signaling pathways and isoforms in genitourinary development become apparent (29).

To delineate the RET signaling pathways critical for the formation of the PNS, we characterized the ENS and ANS in 10 different mutant mice in which key RET docking sites were mutated in both the RET9 and RET51 isoforms. We found that either RET9 or RET51 could support normal ENS development, contrary to a previous report (28); however, dramatic differences were observed when individual phosphotyrosine docking sites were mutated in the context of the RET9 vs. RET51 isoform. In general, mice deficient in a particular adaptor site in the RET51 context exhibited abnormalities that were less severe than those observed in mice harboring the same mutation in the context of RET9, indicating that the additional Grb2 site (Y1096) in RET51

provides redundancy in both the ENS and PNS. For example, ENS and parasympathetic ganglia showed minimal deficits in mice expressing RET51 docking site mutants, whereas mice expressing comparable RET9 mutations showed varying degrees of intestinal aganglionosis and agenesis of key parasympathetic ganglia as well as sympathetic nervous system defects. The phenotypes of these RET mutants (singly or in combination) resemble HSCR and/or CAKUT, including the characteristic incomplete penetrance and variable expressivity of these syndromes. By systematically examining the role of each adaptor singly or in combination with the absence of the Y1096 Grb2-binding site, these studies provide valuable insights into the mechanistic basis for the variable phenotypes observed in HSCR or CAKUT.

\section{Results}

Parasympathetic, but not enteric or sympathetic, ganglia require RET51 isoform for proper development. To investigate the relative contributions of the 2 major RET isoforms, RET9 and RET51, in PNS development, we studied mice that exclusively express 2 copies of WT human RET9 or RET51. These knockin mice, and each of the homozygous $R E T$ mutants studied, were generated previously using homologous recombination of human RET cDNAs into the endogenous Ret locus (knockout-knockin approach) (Figure 1 and Table 1) (29). All analyses performed in this study were on homozygous mice. For PNS analyses, we selected structures that are severely affected in Ret- or GFL-null mice, including the ENS and the cranial parasympathetic sphenopalatine ganglia (SPG) and superior cervical sympathetic ganglia (SCG) of the ANS (9, $10,19)$. Most of the analyses were performed on newborn mice

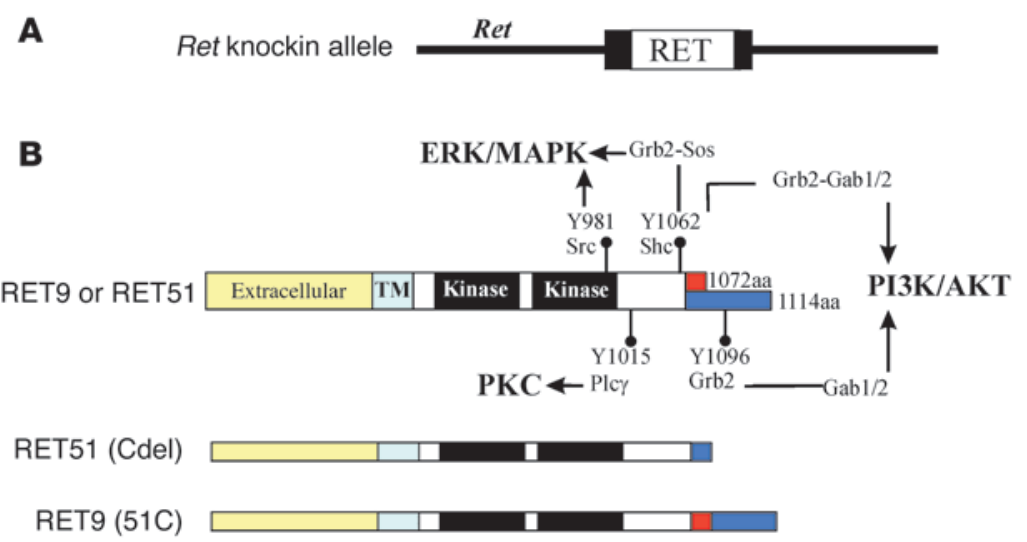

\section{Figure 1}

Schematic of the various $R E T$-mutant mice used in this study. (A) Simplified diagram of mutant or WT human RET cDNAs homologously recombined into exon 1 (black box) of the mouse Ret locus using knockout-knockin approach (29). (B) Schematic of the different WT RET9, RET51, and their respective mutant knocked in alleles is shown. The different domains and sizes of RET9 and RET51 are indicated. The area of divergence between the 2 RET isoforms is indicated in red or blue. Also indicated are the key docking tyrosine $(\mathrm{Y})$ residues, the major intracellular adapters that dock at these tyrosines, and the downstream signaling cascades. Homozygous mice were generated that harbor Tyr-to-Phe (Y to F) mutations for each of the indicated Tyr except Y1096. In the RET51(Cdel) allele, residues 1063-1072 of RET9 were replaced with residues 1063-1072 of RET51. This results in a receptor that is essentially RET51 with a deletion of residues 1073-1114, including Y1096. In the RET9(51C) allele, residues 1063-1072 of RET51 were replaced with residues 1063-1072 of RET9. This results in a receptor that is essentially similar to RET9 with C terminus of RET51, including Y1096. Note Grb2 can directly bind to RET51 and also indirectly to Y1062 in both RET9 and RET51. 

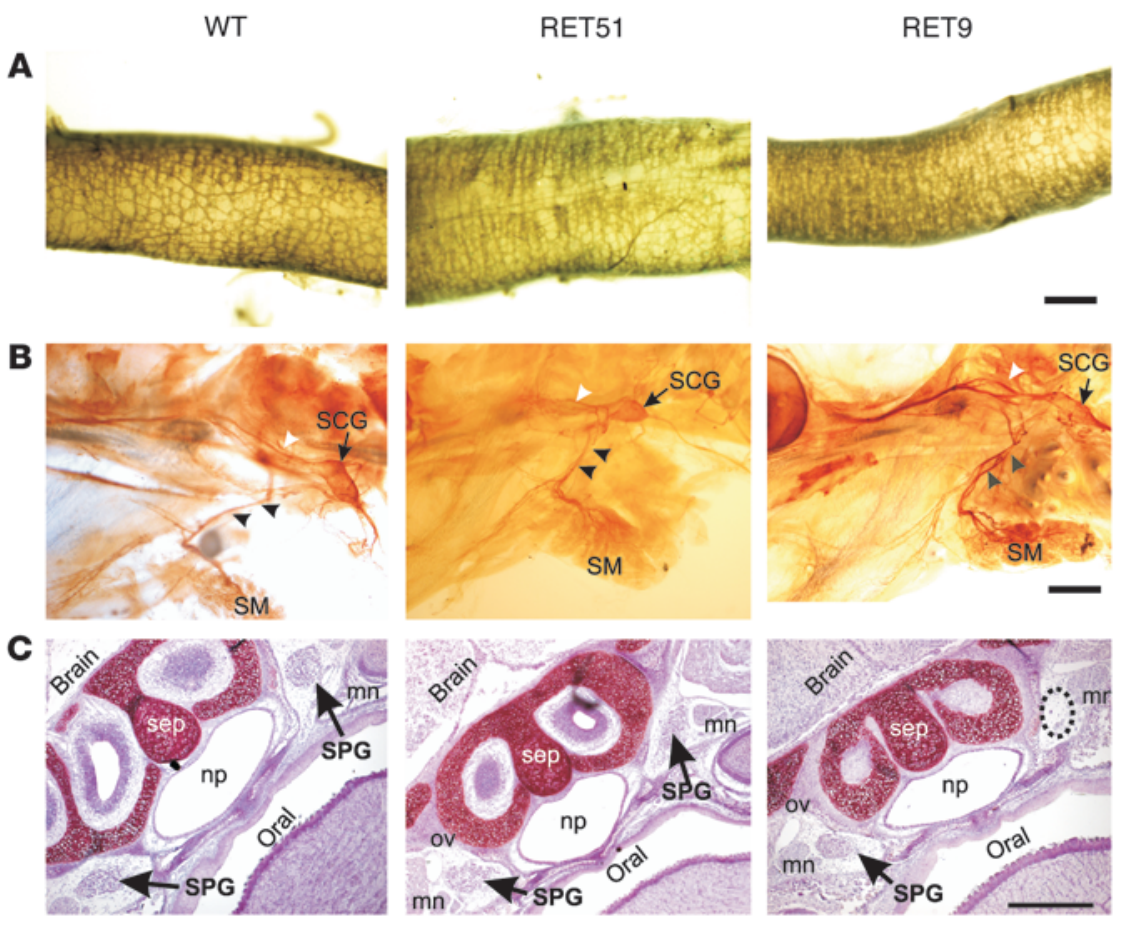

D
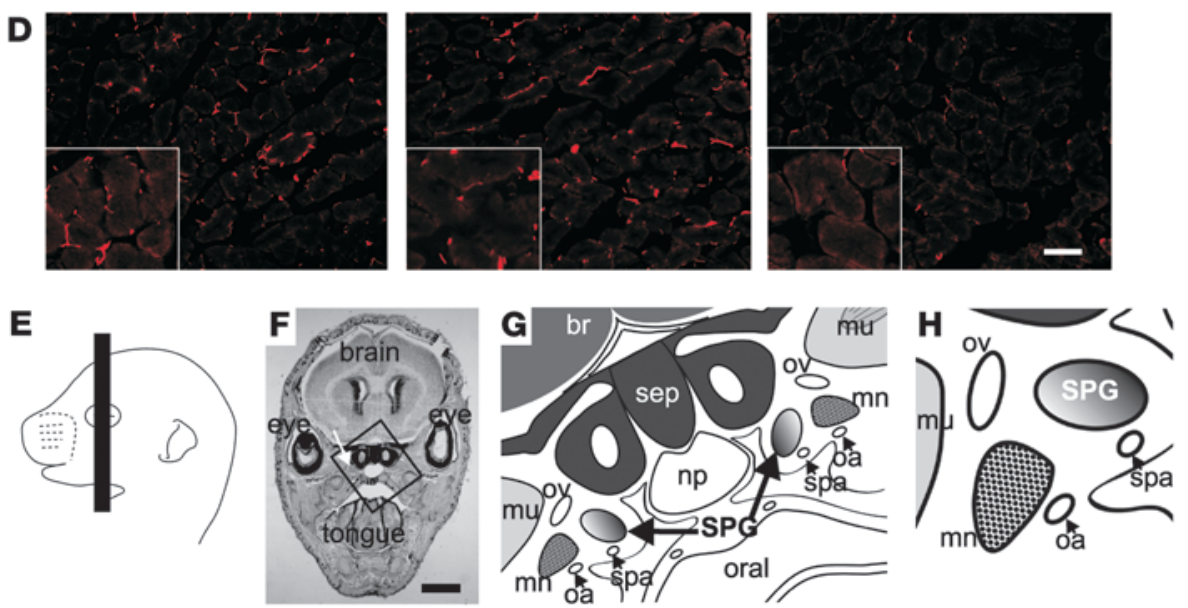

Figure 2

RET51 is sufficient to support ENS and sympathetic nervous system development and is necessary for the development of cranial parasympathetic ganglia. (A) RET9 and RET51 can both support normal ENS development. Whole-mount AChE staining (to visualize ENS formation) from WT or the indicated RET51 and RET9 mice (at postnatal day zero, P0) show typical dense reticular, honeycomb-like distal colon staining, indicating normal ENS colonization by both RET isoforms. (B) Redundant roles of RET isoforms in development of sympathetic ganglia. Whole-mount TH immunohistochemistry (brown color) shows normally located SCG and normal projections to the eye (white arrowhead) and submandibular gland (SM, black arrowheads). (C) RET9 alone is unable to fully support parasympathetic ganglia development. Nissl-stained sections of head from PO mice show normal location of SPG in WT and RET51 (arrows) mice, but a subset of RET9 mice show unilateral SPG agenesis (dashed oval) (see E-H for orientation). (D) Reduced harderian gland innervation in RET9 mice (1- to 2-month-old mice) was observed with anti-TuJ1 immunohistochemistry (red fibers). While TuJ1-positive fibers surround almost all acini in WT and RET51 mice, RET9 harderian glands show reduced innervation consistent with incompletely penetrant unilateral agenesis. (E-H) Anatomical and histological landmarks for SPG analysis. Vertical bar in $\mathbf{E}$ shows approximate level of histological sections. (F) Coronal section showing SPG location (white arrow); boxed area is shown at higher magnification in $\mathbf{G}$, which also represents images in $\mathbf{C}$ and Figure 7A. (H) Closer view of SPG of one side denotes approximate representation of images in Figure 7B. Scale bars: $400 \mu \mathrm{m}$ (A); $600 \mu \mathrm{m}$ (B); $200 \mu \mathrm{m}$ (C); $100 \mu \mathrm{m}$ (D); $50 \mu \mathrm{m}$ (inset); F, $1 \mathrm{~mm}$. br, brain; sep, septum; mu, eye muscles; np, nasopharynx; mn, maxillary nerves; oa, orbital artery; ov, orbital vein; spa, sphenopalatine ganglion.
(P0), as these structures are largely developed at birth, and many of these mutant animals die shortly after birth due to severe renal (29) or intestinal abnormalities (see below).

To determine whether the intestinal aganglionosis in Ret-null mice could be attributed to a specific Ret isoform, we used acetylcholinesterase (AChE) staining to visualize the ENS in mice

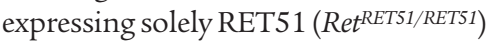

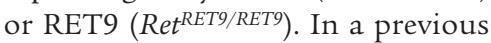
report, Ret9 was found to be critical for intestinal innervation (28); however, we found that either isoform was capable of supporting ENS development. AChE-stained neurons and a normal myenteric neuronal plexus were detected throughout the small and large bowel in these mutant mice (Figure 2A; data not shown).

Previous analysis of Ret-null and Artemin-null mice showed that Ret signaling is important for sympathetic neuron precursor migration and axonal projection $(10,30)$. In these mutants, the SCG fails to migrate to its proper location, resulting in accompanying abnormalities in projections to the submandibular gland and muscles of the eye. The stellate and sympathetic chain ganglia and their axonal projections are also abnormally developed in these mutant mice. We next searched for isoform-specific differences in sympathetic nervous system development. We used tyrosine hydroxylase $(\mathrm{TH})$ whole-mount immunohistochemistry to visualize the sympathetic nervous system in homozygous RET9 and RET51 mice. We found that it developed normally, with properly located SCGs and normal innervation of its targets and normally formed sympathetic chain (Figure 2B and data not shown).

Finally, we investigated to determine whether RET9 and RET51 mice were able to support parasympathetic ganglia development. The SPG, which innervates the harderian/lachrymal glands, fails to develop in Ret-null mice due to defective precursor proliferation (9). Histological analysis of P0 head sections using Nissl staining revealed normally localized SPGs in RET51 mice (Figure 2C). However, a subset of RET9 mice had unilateral agenesis of the SPG (25\%), which was accompanied by reduced harderian gland 

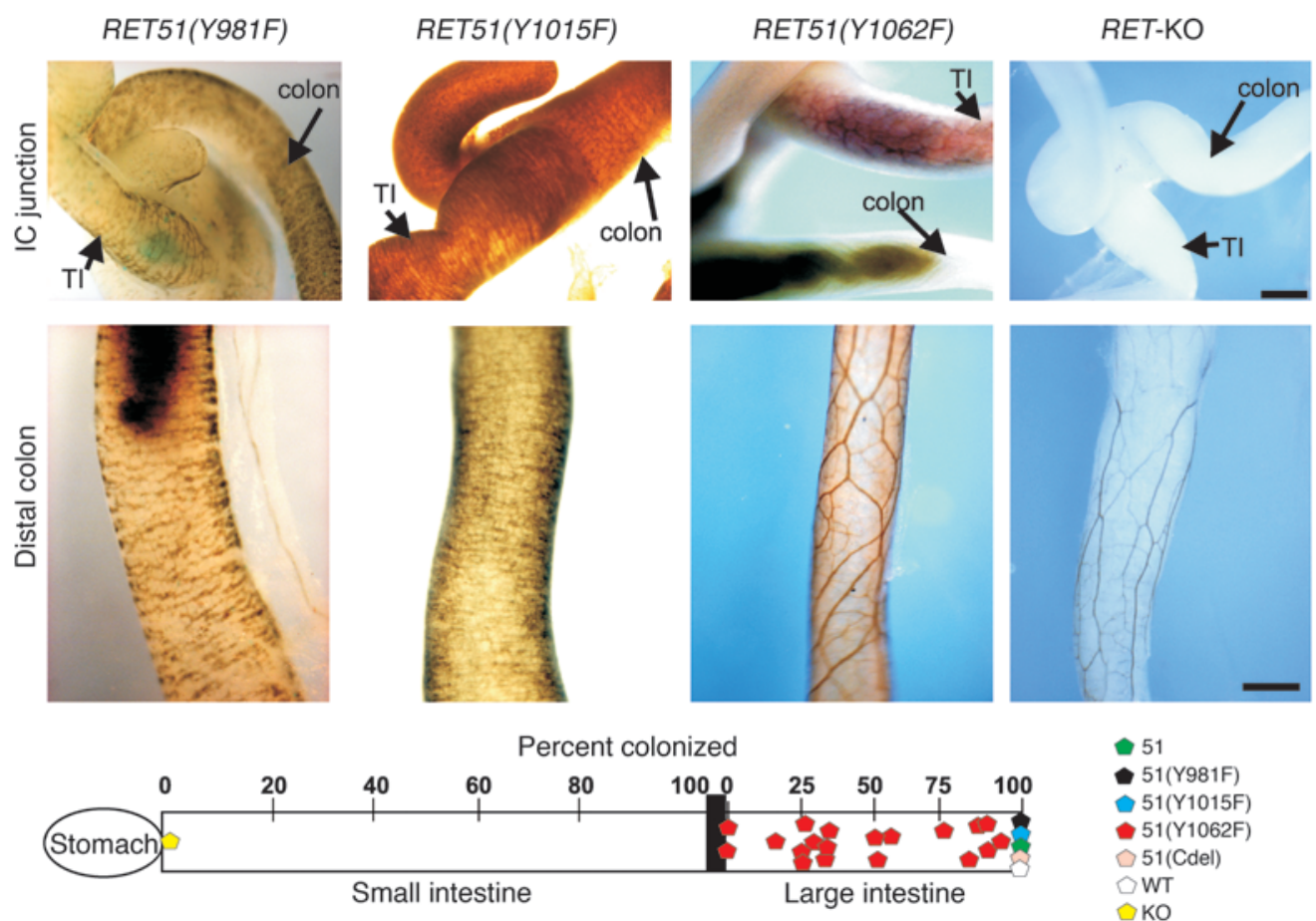

\section{Figure 3}

Essential role of the RET-Shc multidocking site in distal colon innervation. To determine roles of individual RET adaptor sites in ENS development, AChE whole-mount staining to visualize the ENS was performed on intestines (P0) of the indicated adaptor mutants in the context of RET51 isoform (Y981F, Src adaptor; Y1015F, Plcy adaptor; Y1062F, Shc adaptor; or null, KO). Neuronal plexus and ganglion formation, depicted by typical reticular, honeycomb like staining pattern at ileocecal (IC) junction and distal colon, occurs in all adaptor mutants except for RET51(Y1062F). The RET51(Y1062F) image shows distal colon with absent enteric ganglia and plexus, but readily visible thick extrinsic nerve fiber bundles similar to those observed in Ret-null mice. Varying degrees of colon aganglionosis were seen in RET51(Y1062F) mice. Compared with aganglionosis in terminal ileum (TI) of Ret-KO mice, AChE staining of the intestines in RET51(Y1062F) mice detected both a neuronal plexus and ganglia in the TI. The schematic summarizes the extent of bowel colonization by ENS precursors in mutant mice. For RET51(Y1062F), each pentagon represents the location of the most distal enteric ganglion cell in an individual mutant; for other mouse lines, single pentagons represent the entire group, since none of these mice had bowel aganglionosis (refer to graph in Figure 4B for number of mice analyzed for each mutant mouse and associated ENS abnormality). Scale bars: $600 \mu \mathrm{m}$ (IC junction); $400 \mu \mathrm{m}$ (distal colon).

innervation compared with WT mice as assessed by TuJ1 immunohistochemistry (WT $=10821 \pm 977, n=3$; RET9 $=2040 \pm 297$, $n=3$; mean innervation area pixel $^{2} \pm$ SEM, $\left.P<0.001\right)$; innervation in RET51 and WT harderian glands were similar $($ RET51 $=8862 \pm 661$, $n=3$, mean $\pm \operatorname{SEM}, P=0.1$ ) (Figure 2D). These results indicate that the WT forms of RET9 and RET51 have redundant roles in morphologically normal ENS and sympathetic nervous system development. However, RET9 alone is insufficient to promote proper SPG development, suggesting that Grb2-mediated signaling through Y1096 is important in this process.

Signaling through RET51-Y1062 is essential for distal colon innervation. Four phosphotyrosines in the RET cytoplasmic domain serve as major docking sites for intracellular adaptors that initiate downstream signaling cascades. We next sought to delineate which of these RET docking sites, Y981 (site for Src interaction), Y1015 (site for Plcy interaction), Y1062 (interacts with Shc and other intracellular adaptors) and Y1096 (a site only present in RET51 that interacts with Grb2), play critical roles in the ENS in order to gain new mechanistic insights into RET-mediated HSCR.

We used AChE staining to examine the ENS of mice expressing human RET51 in which each of these docking sites was individually mutated (Y to F mutants) or, in the case of Y1096, by using a C-terminal deletion mutant (RET51Cdel) (Figure 1 and Table 1) that lacks this residue $(25,26,28,29)$. We observed remarkable differences in colonic ENS structure in these mutants, which were highly reminiscent of long (aganglionic segment proximal to the rectosigmoid colon) and short segment (aganglionosis confined to rectosigmoid region) HSCR. The enteric plexus and neurons in the distal bowel were absent (i.e., aganglionosis) in mice expressing the RET51(Y1062F) mutant (lacks Shc adaptor site), with the length of aganglionic segment varying from the distal colon only to total colon aganglionosis (Figure 3). Notably, these mice do not have significant renal abnormalities (29), but do not survive to adulthood due to complications of the HSCR-like phenotype (i.e., megacolon, ruptured bowel). Thus, abrogating RET-Shc-mediated signaling has a severe effect on the ENS, but not on urogenital development.

Further, we found that RET51(Y1015F) mice (lack RET-stimulated $\mathrm{Pl} \gamma$ signaling) do not have major abnormalities in the ENS in contrast, with the crucial importance of this pathway in supporting normal urogenital development (29). The majority of these mutant mice showed normal distal colon innervation (13/16), with only a small subset manifesting distal colon hypoganglionosis (Figure 3 and Figure 4B). We also found normal intestinal innervation in RET51(Cdel) mice (RET51 C-term deletion, which lack the direct Grb2 binding site Y1096), RET9(51C), or RET51(Y981F) (lack Src binding site) (Figure 3, Figure 4B, and data not shown). The pheno- 
A
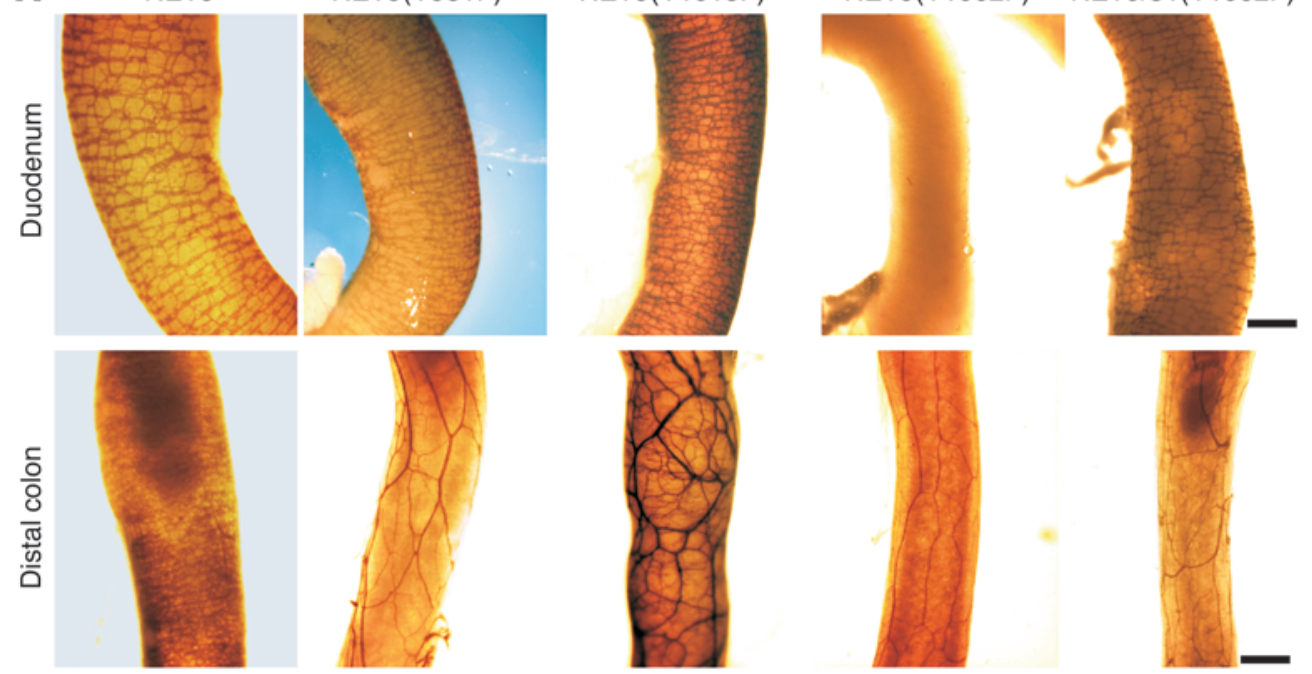

\section{$\mathbf{B}$}

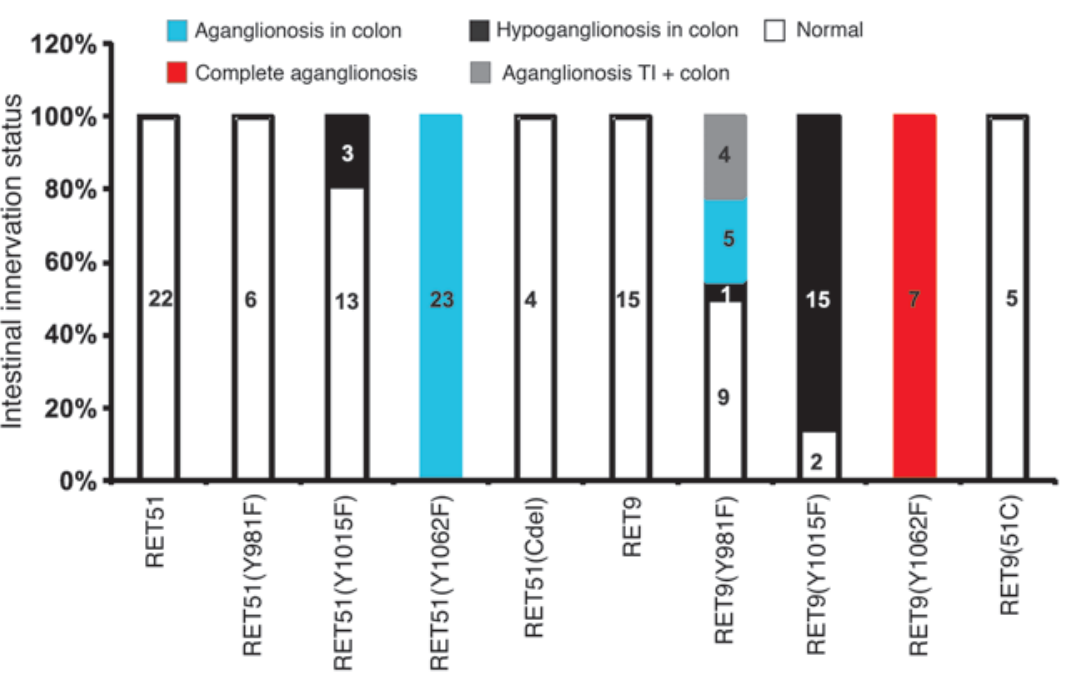

Figure 4

Severe ENS defects in RET tyrosine docking mutants lacking the Grb2-binding site. (A) Whole-mount AChE staining was used to visualize the ENS in PO intestines in RET9 or the indicated RET9 docking tyrosine mutant mice. Regions of the bowel from the distal colon and duodenum are shown. WT RET9 monoisoformic animals have a normal-appearing dense reticular staining pattern in the duodenum and the distal colon, while RET9(Y981F) (Src mutants) have colon aganglionosis but normal duodenum staining. RET9(Y1015F) (Plc $\gamma$ ) mice have neuronal ganglia and plexus in the colon but at reduced density (hypoganglionosis); duodenum innervation is normal. RET9(Y1062F) mice manifest complete intestinal aganglionosis, as no neurons are present in colon or in duodenum. A compound isoformic Y1062F mutant (RET9/51[Y1062F]) has intermediate aganglionosis compared with RET51(Y1062F) (Figure 3) and RET9(Y1062F) mice, indicating isoform dosage influences intestinal innervation. (B) The bar graph summarizes the innervation phenotypes of all the RET9 and RET51 WT and adaptor mutants examined. Numbers in the bars represent the number of mice with that phenotype. Scale bars: $600 \mu \mathrm{m}$ (duodenum); $400 \mu \mathrm{m}$ (distal colon).

types of these mutant mice indicate that in the context of RET51, the Src adaptor site, Grb2-binding site, and the C-term tail are largely dispensable for normal ENS (Figure 4B and Table 2) as well as renal development (29). Furthermore, these results demonstrate that the ENS and genitourinary (GU) systems have different thresholds to the loss of RET-Plcy (results in CAKUT) and RET51-Shc (results in HSCR) mediated signaling for normal development.

Docking site mutations in RET9 cause severe ENS defects. The RET9 isoform differs from RET51 in that it lacks the C-terminal tail that contains the Grb2 docking site (Y1096), important for activation of the AKT/MAPK pathways. This site provides redundancy to the Src and multidocking Shc sites for normal kidney development, as its absence in several of the RET9-Tyr mutants leads to increased severity of kidney abnormalities (29). Therefore, we explored whether this site was also important in ENS formation. AChE staining of postnatal gastrointestinal tracts from mice expressing RET9 mutations (Y981F, Y1015F or Y1062F) revealed a spectrum of phenotypes that were more severe than those of mice expressing the corresponding RET51 mutations. For example, while the ENS of RET51(Y981F) mutant mice appeared normal, RET9(Y981F) mice manifested partially penetrant distal intestinal aganglionosis $(9 / 18)$ in which the extent of aganglionosis ranged from the distal third of the colon only to the entire colon and terminal ileum (Figure 4). Interestingly, the RET9(Y981F) mutant mice previously showed the pattern of 


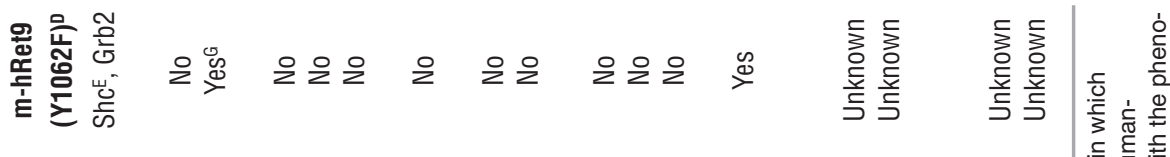

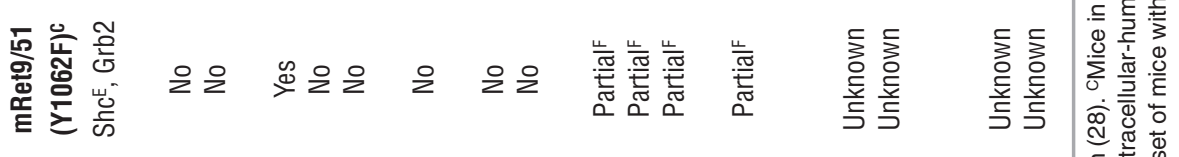

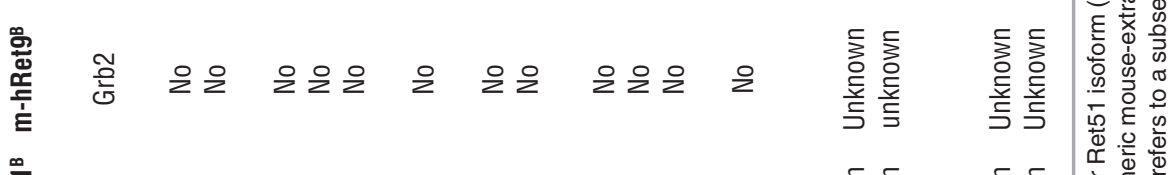

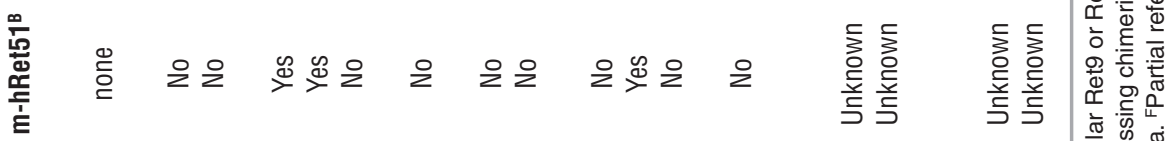

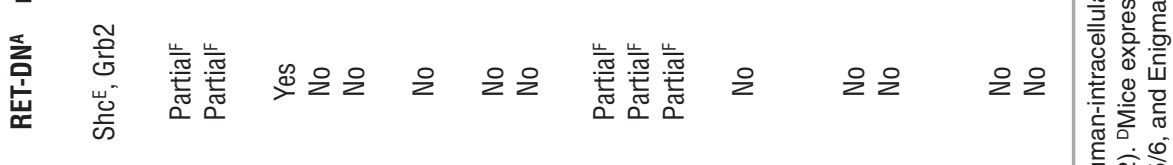

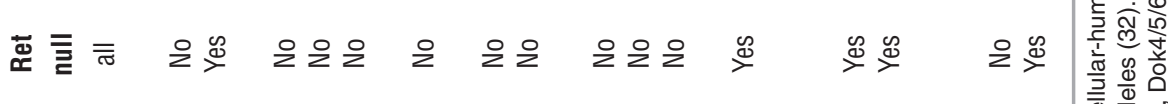

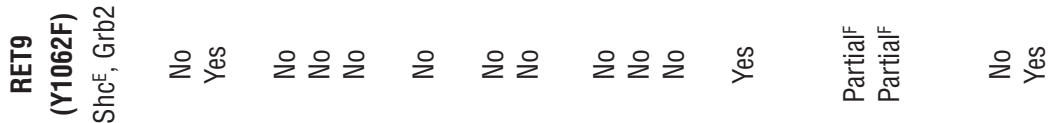



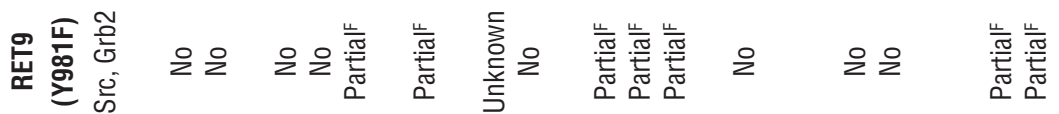
0 N

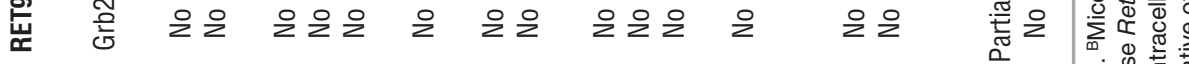

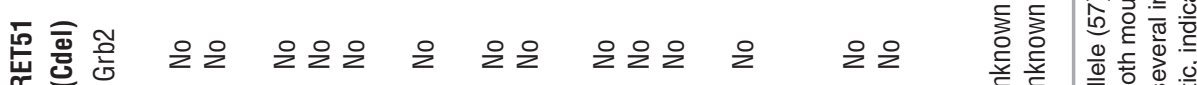

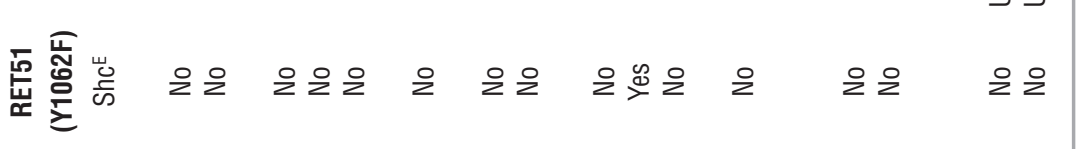

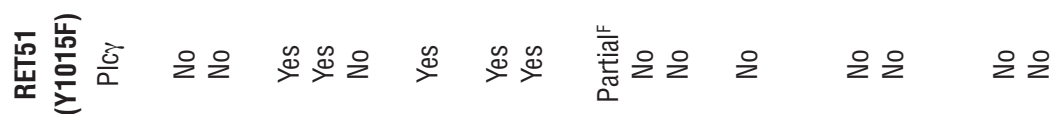

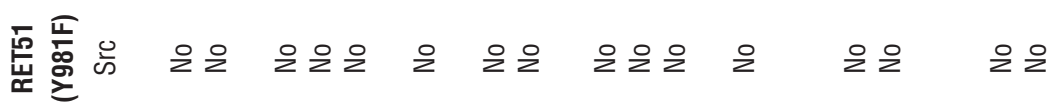

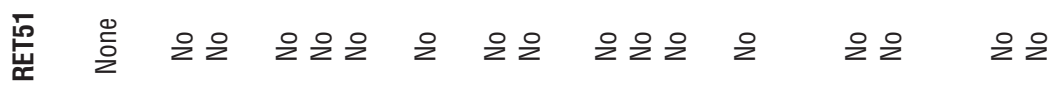

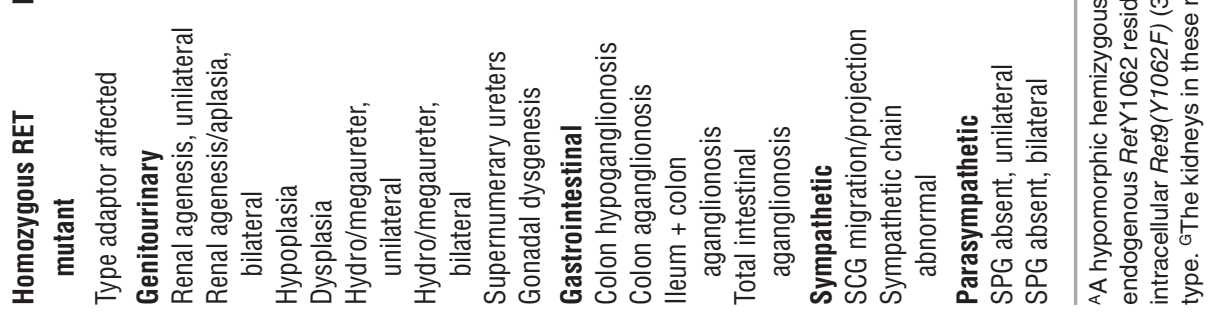



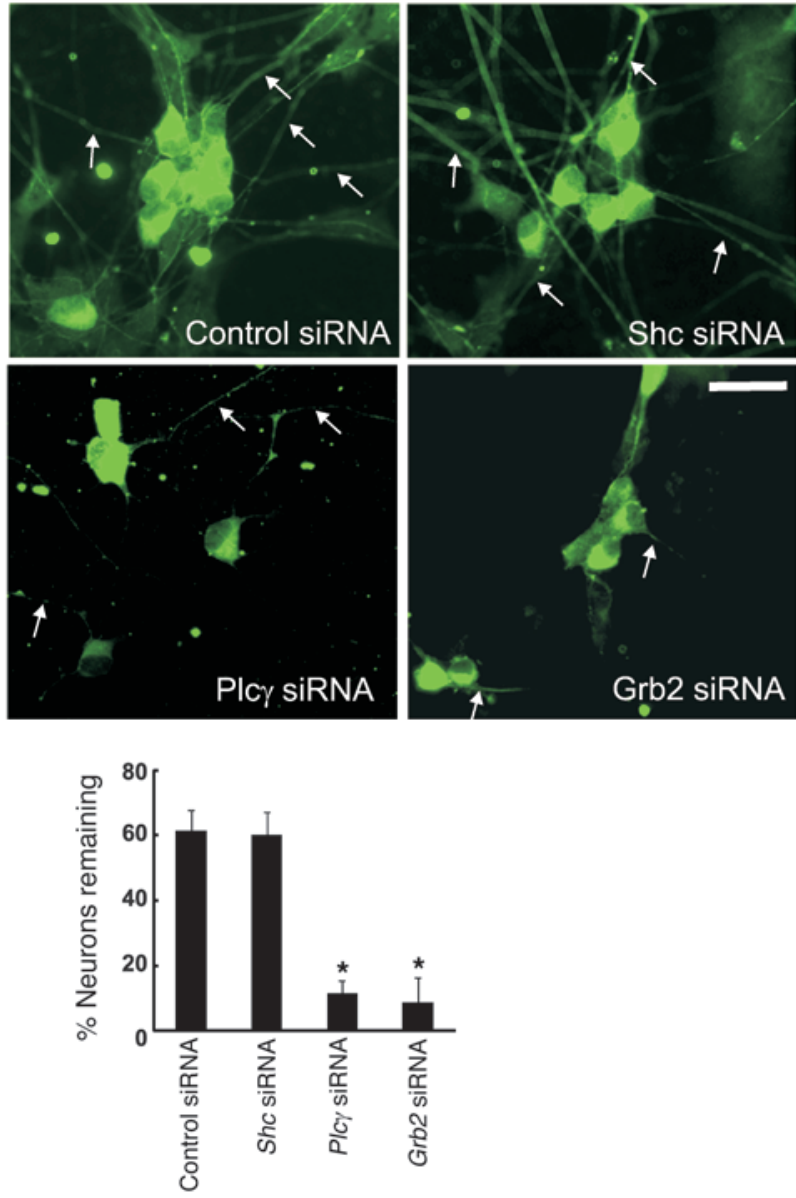

incomplete penetrance and variable expressivity in CAKUT (29) (Table 2). Thus, abrogated Src adaptor site binding in the face of reduced Grb2-mediated signals (i.e., RET9 context) increases the propensity to develop both HSCR and CAKUT phenotypes.

In contrast with the abnormalities in the RET9(Y981F) mutants, RET9(Y1015F) mutants (lack Plcy binding) have normal-appearing small intestinal innervation and show only a partially penetrant distal colon hypoganglionosis (15/17) (Figure 4, A and B). While of moderate severity, these colon ENS abnormalities are significantly worse than those observed in RET51(Y1015F) mice. We noted that the differences in penetrance and severity of ENS deficits between RET9-Plcy and RET51-Plcy mutants (Figure 4) were not observed in the genitourinary system in which both mutants manifested severe genitourinary defects (29) (Table 2). We also examined the ENS of RET9(Y1062F) mice (lack binding to Shc and other adaptors). We found that these mice have complete intestinal aganglionosis with $100 \%$ penetrance, a phenotype that is similar to Ret-null and mouse-human-chimeric-Ret9(Y1062F) mutant mice $(19,31)$. These deficits were more severe than in RET51(Y1062F) mutant mice analyzed above in which aganglionosis is limited to the colon and further support the idea that Grb2 signaling via the RET51specific Y1096 docking site is important for proper development of the ENS. Finally, compound isoformic RET(Y1062F) mutant

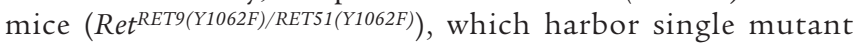
RET9(Y1062F) and RET51(Y1062F) alleles, had an intermediate phenotype, with aganglionosis affecting colon and terminal ileum that is reminiscent of long-segment HSCR (32) (Figure 4A). We

\section{Figure 5}

Grb2 and Plc $\gamma$ are essential for maintaining normal enteric neuron number. Roles of Grb2, Plc $\gamma$, and Shc adaptor proteins in regulating enteric neuron number were examined in vitro. ENS precursors were harvested from E14.5 rat intestines using p75 ${ }^{\mathrm{NTR}}$ immunoselection and propagated in culture for 7 days. Indicated siRNAs or control (scrambled siRNA) were delivered by lentivirus infection 1 day after plating the cells. Tau1 immunofluorescence was used to detect neurons and their neurites (arrows). Shc siRNA did not affect cell number compared with control. PIc $\gamma$ and Grb2 siRNA caused a severe reduction in neuron numbers. The $y$ axis represents neurons remaining relative to the beginning of the culture. The graph at the bottom shows quantification results from 3 independent experiments (mean $\pm \mathrm{SD},{ }^{*} P<0.05$ versus control). Scale bar: $25 \mu \mathrm{m}$.

did not observe any overt differences between males and females for aganglionosis or hypoganglionosis phenotypes in the mutant mice analyzed above. Taken together, these results suggest that activation of AKT and MAPK signaling through Y1062 that serves as a docking site for Shc and other adaptor proteins is necessary for complete colonization of the colon.

To further determine the importance of Grb2-mediated signaling in ENS development, we isolated and cultured ENS precursors from E14.5 WT rat bowel using P75 immunoselection (see Methods). We infected these with lentiviruses expressing siRNAs directed against either Grb2, Shc, or Plcy and performed analysis using TuJ1 and Tau1 immunocytochemistry (33) (see Methods). Grb2 knockdown resulted in a marked reduction in enteric neuron number compared with control or Shc siRNA-expressing cells, further confirming the essential role of Grb2 in ENS development; both Tau 1 and TuJ1 immunocytochemistry revealed similar reductions in neuronal number (Figure 5 and data not shown). The fact that $S h c$ knockdown did not have a major effect on enteric neuron number suggests redundancy/compensation through other adaptors and is consistent with milder ENS phenotype observed in RET51(Y1062F) mutant mice, which harbor the additional Grb2binding site. Interestingly, Plcy knockdown also caused a dramatic reduction in neuronal numbers despite the minor ENS deficits in $R E T(\mathrm{Y} 1015 \mathrm{~F})$ mice, suggesting that Plc $\gamma$ activation via signals other than RET may be important in ENS development.

Sympathetic nervous system development is relatively resistant to mutation of individual RET docking sites. Artemin-stimulated RET signaling is important for sympathetic neuron precursor migration and subsequent axonal projection. To decipher RET-dependent pathways that govern sympathetic ganglia formation, we used wholemount TH immunohistochemistry to visualize the sympathetic nervous system in these RET-mutant mice. We found that individual docking sites for Plcy, Src, Shc, and Grb2 in RET51 isoform are all dispensable for sympathetic nervous system development (Figure 6, Table 2, and data not shown). When we examined mice expressing RET9 with these mutations, we again found few deficits in the sympathetic nervous system. These included moderate, unilateral abnormalities in SCG migration, sympathetic chain development, and neuronal projection defects in a subset of RET9(Y1062F) mutant mice (5/8) (Figure 6). These results are in stark contrast to the deficits observed in the ENS and in kidney development in these mice (Table 2), suggesting that sympathetic nervous system development has a different threshold to aberrant Ret-Tyr signaling than other systems or depends on a high degree of Ret-mediated signaling redundancy. 

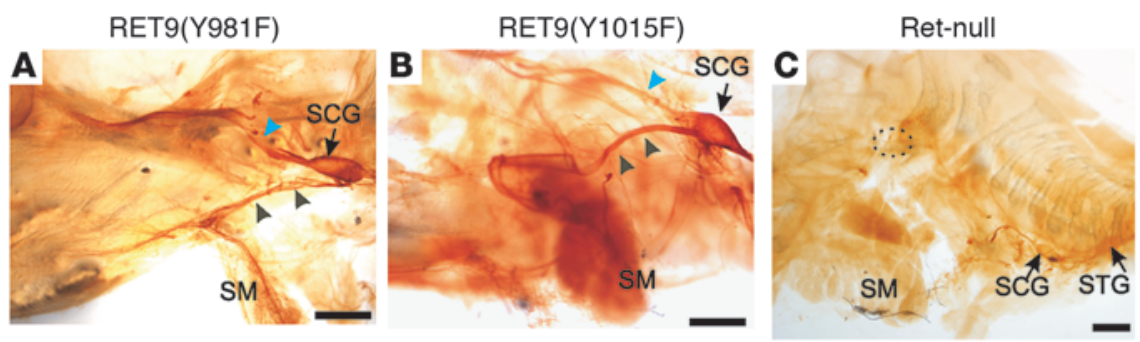

RET9(Y1062F)
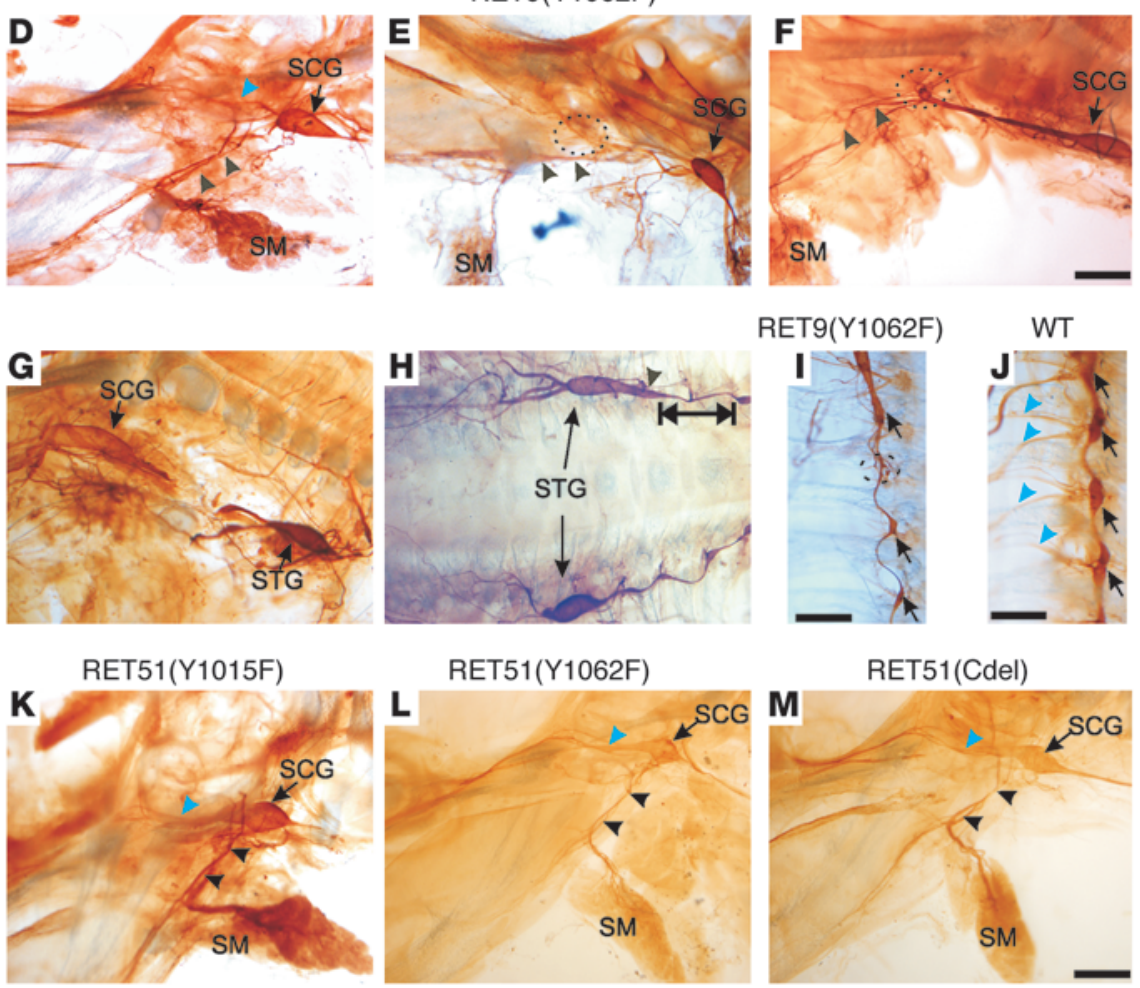

\section{Figure 6}

Sympathetic nervous system defects in RET mutants lacking Shc and Grb2 binding sites. Whole-mount TH immunohistochemistry was performed on PO pups to assess sympathetic nervous system development in RET9 and RET51 signaling mutants. (A-C) Normal sympathetic nervous system develops in (A) RET9(Y981F) (Src mutant) and (B) RET9(Y1015F) (PICY mutant) mice, as demonstrated by normal SCG location and normal innervation to submandibular gland (SM, black arrowheads) and the eye (blue arrowhead). (C) Ret-null mice show abnormal caudal location of the SCG, next to the stellate ganglion (STG) instead of the expected normal location (dashed oval); innervations to the SM or the eye are absent. (D-H) RET9(Y1062F) adaptor mutants (lack Shc- and Grb2-binding sites) show a spectrum of sympathetic nervous system defects albeit milder than Ret-null animals. These include normal SCG location and projections to eye (blue arrowhead) and submandibular gland (black arrowhead in D), failure of SCG to migrate normally ( $E$ and $\mathbf{F}$; dashed circle depicts expected location), mislocalization of the SCG near the stellate ganglion (STG) in G, and fusion of rostral sympathetic chain ganglia (black arrowhead) with the STG, resulting in a gap in the sympathetic chain (double arrowhead) in $\mathbf{H}$. (I) Sympathetic chain (sc) ganglia in RET9(Y1062F) mice are small (black arrows), or absent (dashed oval) and have diminished to absent axonal outgrowths compared with WT mice (blue arrows) in $\mathbf{J}$. (K-M) Docking site mutations in the context of RET51 do not disrupt sympathetic nervous system development. Representative pictures are shown for the indicated adaptor mutants in RET51 context, highlighting normal sympathetic ganglia development. Scale bars: $600 \mu \mathrm{m}(\mathbf{A}-\mathbf{H}$ and $\mathbf{K}-\mathbf{M}) ; 400 \mu \mathrm{m}(\mathbf{I}, \mathbf{J})$.
Severe parasympathetic nervous system defects in RET9 docking tyrosine mutant mice. In all the RET-dependent organs examined (ENS, sympathetic nervous system, kidneys), RET9 docking site mutants (lack Grb2 binding at Y1096) manifest more severe defects than mice expressing their RET51 counterparts. To extend this analysis to the parasympathetic nervous system, we examined SPG development in these mutant mice. The SPG failed to develop in a large number of RET9-Tyr mutants, and these were generally more severe than mice expressing WT RET9 (Figure 2C and Figure 7). For example, RET9(Y981F) (lack Src binding) and RET9(Y1015F) (no Plcy binding) mutants showed partially penetrant unilateral and bilateral SPG agenesis, with accompanying reduced innervation to the harderian gland in the postnatal period (Figure 7, A-C and E). Importantly, RET9(Y1062F) mutants (no Shc or Grb2 binding) have completely penetrant bilateral SPG agenesis, as observed in Ret-null mice (Figure 7A). We counted the number of Nissl-stained neurons in these mutant mice using serial sections of the entire SPG and found that when the SPG is present (i.e., no agenesis), the ganglia contained a normal number of neurons (Figure 7D). Notably, histological analysis of P0 head sections using Nissl staining revealed normal localization of SPGs and normal neuronal numbers in all mutants in the RET51 context (Figure 7 and data not shown). More severe SPG defects in RET9 mutants again indicate that Grb2 signaling from RET51 C terminus is important for normal development of the parasympathetic nervous system.

\section{Discussion}

Inactivating RET mutations are the most commonly identified cause of human HSCR and have recently been implicated in renal agenesis $(3,7)$. Activating RET mutations cause MEN2A and MEN2B, familial cancer predisposition syndromes affecting the adrenal medulla, parathyroid, and thyroid (34). Many of these mutations are in the cytoplasmic domain of RET. The activation of major signaling proteins such as PLC $\gamma$, SRC, PI3K, and MAPK by GFL signaling depends on key RET docking tyrosines for intracellular adaptors. One poorly understood aspect of these disease associations is the molecular mechanisms that underlie organ- or tissue-specific effects of RET mutations in human disease and the role of these RET-activated pathways in organogenesis. Through the analyses of mutant RET allelic series, new insights into the role of RET isoforms and signaling pathways in the ENS and ANS development as well as congenital diseases caused by RET mutations have been made. In particular, through our comparative analyses, we found remarkable specificity in the signaling systems required for each region and demon- 
A

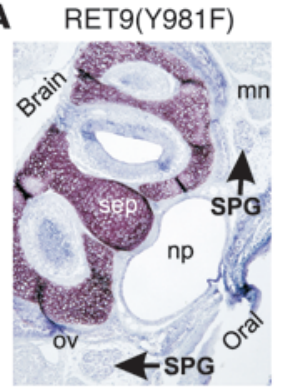

RET9(Y1015F)

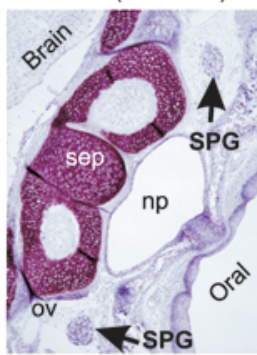

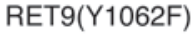



RET51(Y1062F)

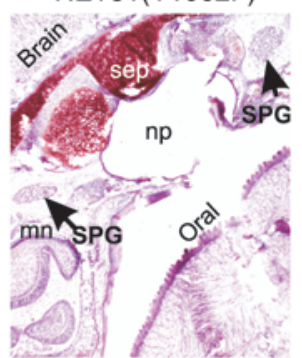

Ret-null

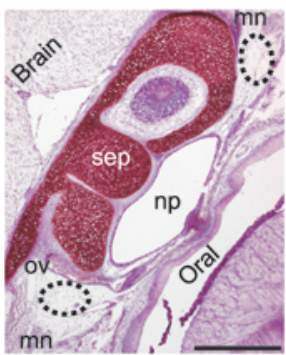

B
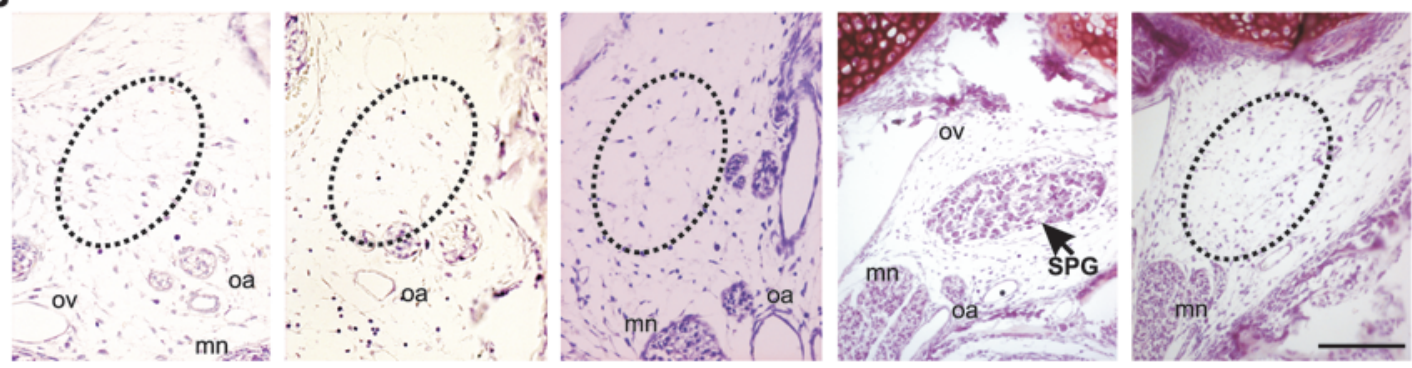

C

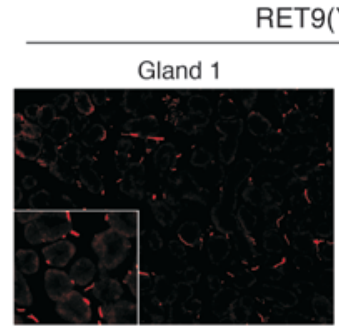

RET9(Y981F)

\section{RET9(Y1015F)}
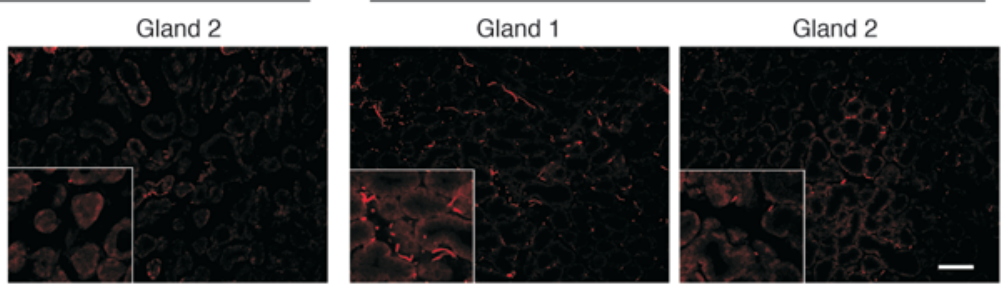

D

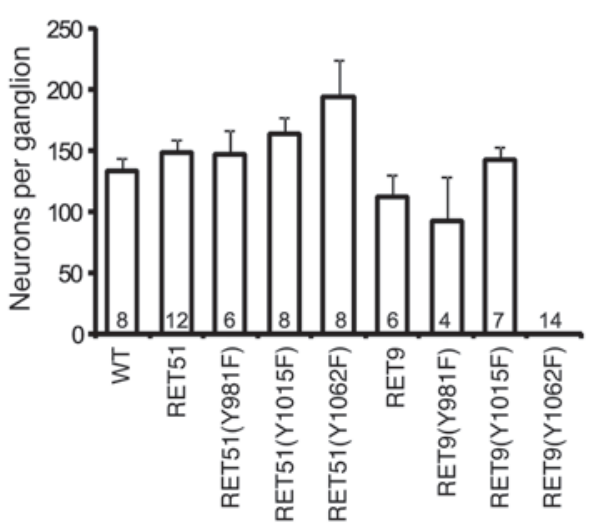

E

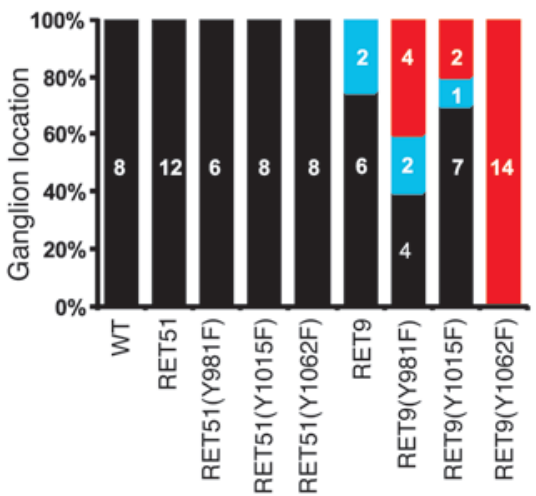

Normal

Unilateral agenesis - Bilateral agenesis

Figure 7

Severe parasympathetic nervous system defects in RET9 docking tyrosine mutant mice. Cranial parasympathetic SPG and their innervation of the harderian gland using Nissl staining (P0 pups) and TuJ1 immunohistochemistry (1- to 2-month-old mice), respectively. Refer to Figure 2, $\mathrm{E}-\mathrm{H}$, for anatomical landmarks for SPG and key for annotations. (A and B) RET9(Y1062F) mice exhibit $(n=14)$ bilateral SPG agenesis, a phenotype similar to that of Ret-null mice (dashed oval, expected normal SPG site); RET51(Y1062F) SPG were normally located (arrows in A and B). RET9(Y981F) and RET9(Y1015F) mice showed incomplete penetrance of SPG agenesis, including normal SPG location bilaterally (arrows in A), unilateral, or bilateral SPG agenesis (SPG agenesis indicated by dashed ovals in B and summarized in E). (C) Differences in innervations (TuJ1 staining, red fibers surrounding each acinus) of the 2 harderian glands from the same mutant mouse. Note that gland 1 of each mutant had TuJ1-positive fibers surrounding all acini, but gland 2 innervation was markedly decreased or absent, consistent with SPG unilateral agenesis (closer view shown in the inset). (D) Summary of SPG neuronal numbers in RET mutants, in which SPG are formed. Quantification of neuron numbers in SPG show no significant differences in mutant and WT mice except RET9(Y1062F) mice, which had SPG agenesis. The graph depicts number of neurons in each completely sectioned SPG (mean \pm SEM; numbers at the bottom denote SPG used per genotype). (E) Spectrum of SPG abnormalities observed in RET-mutant mice. Results of SPG location from both RET51 and RET9 mutant mice are summarized in the bar graph. Numbers in each colored bar represent the number of SPG with the corresponding defect. Scale bars: $200 \mu \mathrm{m}(\mathbf{A}) ; 50 \mu \mathrm{m}$ (B); $100 \mu \mathrm{m}$ (C); $50 \mu \mathrm{m}$ (inset). 


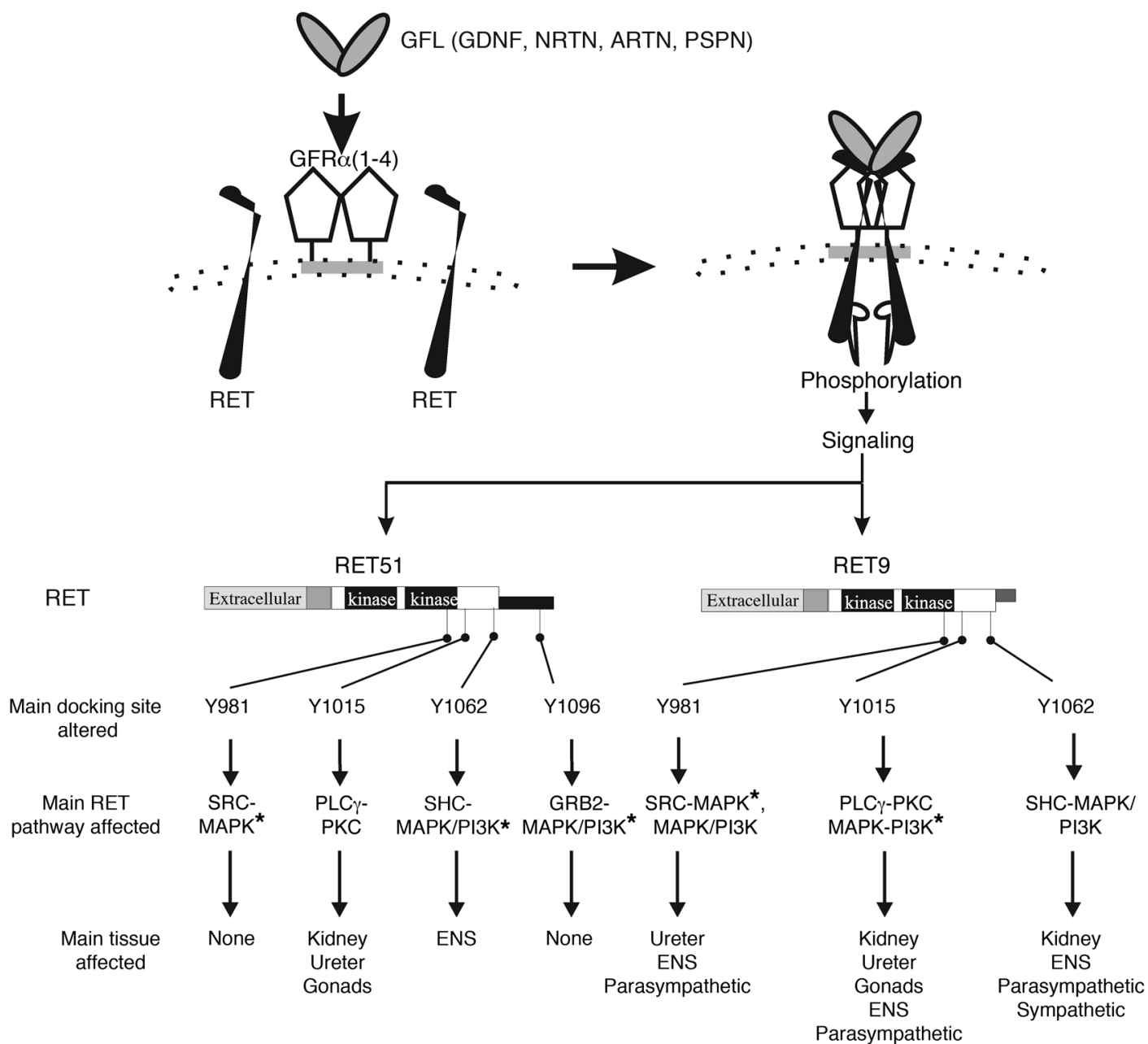

Figure 8

Summary model depicting the main tissues affected by key docking tyrosines activated by GFL-RET signaling. One of the 4 GFLs binds 1 of the 4 GFR $\alpha$ coreceptors and forms a multimeric complex with RET leading to receptor activation. The phosphorylated docking sites on each of the major isoforms, RET9 and RET51, interact with the indicated adaptors and the signaling cascades. RET9 lacks the extra GRB2-binding site (Y1096), and defects in general are more severe and affect more systems when the docking sites are mutated in RET9 context. Asterisks on the MAPK/PI3K pathways denote partial reduction due to redundant activation through intact Y1062 or Y1096.

strated redundancy in RET-dependent signaling for organogenesis, with some mutations affecting the ENS but not renal development, whereas others (Plcy signaling) have more severe effects on kidney formation than on the ENS.

One important aspect of our study is the finding that both the human RET isoforms, RET9 and RET51, support normal ENS and sympathetic nervous system development in mice. Previous in vitro studies have presented contrasting views regarding the relative importance of these isoforms and each has been touted as more crucial $(35,36)$. An additional study regarding the in vivo roles of Ret isoforms concluded that Ret51 could not support normal kidney or ENS development, whereas the formation of these systems was normal in Ret9-expressing mice (28). In contrast, our studies point to redundant roles of the RET isoforms in that we observed the development of morphologically normal kidneys, ENS, and sympathetic nervous system, with only the parasympathetic system demonstrating isoform-specific requirements. The reasons for these differing results are unclear and have been discussed before (29). These include different properties of the chimeric mouse-human Ret51 or Ret 9 proteins used in the de Graaff study (28) that contain mouse genomic region encompassing the extracellular domain versus the cDNAs of the human isoforms used in our study (Table 2). There may also be environment or genetic strain differences that account for our different results. Nevertheless, the observations raise the possibility that $R E T$ isoforms could play alternative roles depending on genetic or environmental contexts.

The availability of mice with individual mutations in each RET isoform enabled us to systematically determine the role of each adaptor site individually in PNS development. In general, RET9 mutations caused more severe abnormalities than comparable mutations in the RET51 isoform (Figure 8 and Table 2). For example, RET51(Y981F) (Src site) or RET51(Y1015F) (Plcy site) did not prevent colonization of the bowel by ENS precursors, whereas these same mutations in the RET9 context resulted in hypoganglionosis $(\mathrm{Y} 1015 \mathrm{~F})$ or aganglionosis $(\mathrm{Y} 981 \mathrm{~F})$ of the distal bowel in a large number of animals. Further, mutations in Y1062 also caused more severe abnormalities in the RET9 context (complete 
intestinal aganglionosis) than in RET51 animals (only distal colon aganglionosis). Each of these observations suggests an important role for Grb2 signaling in ENS development, a hypothesis supported by our Grb2 knockdown experiments with ENS precursor cells and by previous studies in vitro $(35,37,38)$. Similar increases in the severity of observed defects were found in the parasympathetic and sympathetic ganglia, consistent with an important role for Grb2 in GFL-mediated nervous system development. Overall, this increased severity may be due to synergistic effects on PI3K/AKT and MAPK activity in these RET-dependent tissues due to the lack of this additional Grb2-binding site in RET9 mice. Biochemical characterization of PI3K/AKT and MAPK activity in neurons from these mutant animals showed modest reductions in RET9(Y981F) and RET9(Y1015F) mutants and complete abrogation of PI3K/AKT and MAPK activity in RET9(Y1062F) mutants $(29,39)$. These results are consistent with redundancy mediated by Grb2 interactions at Y1062 (via Shc) or direct binding at Y1096 and highlight the differences in RET51 versus RET9 signaling properties in vivo $(35,37,38)$.

Our studies also identify the molecular signaling pathways that lead to the panoply of defects observed in Ret-null mice, including renal agenesis and complete intestinal aganglionosis as well as parasympathetic and sympathetic nervous system defects. However, they also reveal why abnormalities present in the majority of HSCR patients, such as colon aganglionosis, can occur without involvement of other organs. For example, the phenotype of RET51(Y1062F) mice is very similar to that reported for HSCR caused by a RET mutation in the SHC docking site (RET51-M1064T) (40). While the cellular processes defective in mice expressing RET(Y1062F) remain to be definitively identified in vivo, the phenotypes are most likely due to reduced precursor proliferation and migration.

HSCR and CAKUT are complex diseases that can occur singly or together $(7,40)$. The abnormalities in these disorders are often complicated by incomplete penetrance and variable expressivity, thus making it difficult to delineate the underlying pathogenetic mechanisms. RET mutations account for the majority of familial and sporadic HSCR and are also found in many cases of renal aplasia $(4,41)$. Our comparative analyses of the PNS and genitourinary system development (29) in these RET isoform/docking tyrosine mice mutants (Table 2) exhibit remarkable similarities to manifestations of HSCR and CAKUT in humans and provide mechanistic insights into how RET-mediated signaling defects may lead to various manifestations of these diseases. For example, we discovered genetic combinations characterized by similarities to HSCR alone (RET51[Y1062F]), CAKUT alone (RET51[Y1015F]), or to a combination of HSCR and CAKUT (RET9[Y981F] and RET9[Y1062F]). Furthermore, these mutants (e.g., RET51[Y1062F] and RET9[Y981F]) exhibit ENS innervation deficits of varying degrees, a prominent feature of HSCR, which suggests that aberrant signaling through these docking sites underlies the variability in HSCR phenotypes. Notably, mutations within the RET cytoplasmic domain have been identified in patients with HSCR as well as renal agenesis, although to our knowledge not in the key docking tyrosines, as they may be detrimental to life. These include mutations within the cytoplasmic domain (S767R, Y791F, K907E, E921X/K, M980T, M1064T) that affect residues important for kinase activity or autophosphorylation of the key docking tyrosines (Y981, Y1015, Y1062, and Y1096) and that can modulate activation of downstream signaling pathways $(4,7,40,42-46)$. Since these docking tyrosines are the major effectors of GFL-mediated RET activation, these mutant mice pro- vide in vivo information regarding the signaling pathways whose disruption influences the likelihood and severity of disease in patients with CAKUT and HSCR. Importantly, these studies also suggest that the relative expression of the 2 RET isoforms could play a key role in the severity of these diseases, as signaling through Grb2 docking site at Y1096 acts in a synergistic manner with the other RET signaling pathways.

Several important observations from our study provide insights into the basis for organotypic specificity in CAKUT and HSCR and the regulation of PNS and GU development. For example, RETstimulated Plcy signaling (through Y1015) appears to have different roles in GU versus PNS development. In the GU system, RET$\mathrm{Pl} \gamma \gamma$ abrogation results in CAKUT encompassing bilateral defects including gonadal dysgenesis, ectopic ureters, distal obstruction, and renal branching abnormalities secondary to enhanced Ret signaling, likely due to inhibition of RTK repressors such as Spry1 $(29,47)$. However, in enteric and parasympathetic ganglia, cells in which Spry1 and Ret do not interact $(47,48)$, reduced Ret signaling results in reduced ganglia formation. It should be noted that among the cranial parasympathetic ganglia, we limited our analyses to the SPG in this study due to complete penetrance of the SPG phenotype in Ret-null mice and the relative ease of its analysis and analysis of its target, the harderian gland, and thus our results are only relevant to the SPG. Overall in the PNS, tyrosine mutations in the RET docking site appear to reduce the number of neurons and axonal projections. This is consistent with studies of HSCR in which variable aganglionosis is observed regardless of the location of the mutation (41). However, in the GU system, these key docking sites appear to have distinct roles: Plcy in ensuring only a single kidney is formed and PI3K/MAPK in promoting initiation of kidney formation. We also observed that mice expressing RET9 mutations that exhibit incomplete penetrance but affect more than one organ system (i.e., RET9[Y981], RET9[Y1015F]) do not always manifest all the defects, indicating that individual organs have differential susceptibility or thresholds to the same mutation. For example, defects in $R E T(Y 981 F)$ animals include CAKUT with a normal ENS, HSCR with normal kidneys, or CAKUT and HSCR together, detected even in siblings from the same litter. The basis for these organ-type variations is not entirely clear and is most likely due to tissue-specific modifier genes or epigenetic mechanisms.

Mice evaluated in this work have a wide range of defects in the ENS, ANS, and kidneys that mimic human diseases. Although certain RET-mediated signaling pathways appear more important for some tissues than others, most of the RET9 mutations affect at least the ENS, kidney, and parasympathetic nervous systems. This suggests that more thorough evaluations of these systems in children with HSCR or CAKUT might be useful. In fact, a recent study demonstrated that CAKUT anomalies occur in about $25 \%$ of children with HSCR (7). Furthermore, RET mutations have previously been detected in patients presenting with MEN2A in combination with either HSCR or CAKUT (4-7). In particular, tests for parasympathetic nervous system function (e.g., dry eye disease) in individuals with HSCR could be helpful. Finally, our discovery that mice expressing RET(Y1015F), which is the docking site for $\mathrm{Pl} \gamma$, have CAKUT along with distal hypoganglionosis suggests the possibility of subclinical gastrointestinal dysfunction in these patients that may not present as HSCR.

Specificity and redundancy of individual isoforms of a number of other RTKs (e.g., Met, PDGFR, insulin receptor, FGFR1) have an impact on their biological activities $(49,50)$. We report here 
a comprehensive analysis of key signaling pathways activated by GFL-mediated RET activation that identified distinct as well as redundant roles for ENS, ANS, and kidney development.

\section{Methods}

Generation of various RET knockin mice. All animal studies were approved by the Washington University Animal Studies Committee. Human RET9, RET51, and the indicated mutant cDNAs were homologously recombined into the first coding exon of the Ret gene to disrupt synthesis of the endogenous mouse Ret as previously described (Figure 1 and Table 1) (29). All experiments were performed on homozygous mice with a mixed genetic background (129/SvJ:C57BL/6) obtained by breeding of corresponding hemizygous parents.

Histopathological and morphological analysis of the PNS. Mouse tissues were harvested at the indicated ages, fixed in $4 \%$ paraformaldehyde, and processed as previously described (11). Routine histological assessment was done on H\&E-stained tissue sections.

Evaluation of the ENS was done using whole-mount AChE staining on dissected gastrointestinal tract of newborn (P0) or postnatal mice. The extent of aganglionosis was determined as a percentage of large or small bowel that was not colonized by enteric neurons as previously described $(11,51,52)$.

Catecholaminergic sympathetic neurons were visualized with diaminobenzidine by means of whole-mount TH immunohistochemistry (rabbit polyclonal antibody, 1:200; Chemicon) on paraformaldehyde-fixed P0 mouse pups as described (10).

The location and number of SPG neurons was determined from thioninstained (Nissl), paraffin-embedded coronal head sections $(6 \mu \mathrm{m})$ of newborn mice. Since slight variations in embedding/sectioning angle could influence simultaneous appearance of this small ganglion from both sides in the same section, the region encompassing the entire ganglion was sectioned, and neurons with distinct nucleoli were counted at $120-\mu \mathrm{m}$ intervals (at least 3 animals, 6 ganglia, from each genotype) as previously described (11). The histological and anatomical annotation and plane of sections are depicted in Figure 2, E-H.

The innervation of intraorbital harderian gland was examined on cryosections $(10 \mu \mathrm{m})$ of fixed tissue with rabbit class III $\beta$-tubulin antibody (1:500; Covance) and visualized with Cy3-conjugated anti-rabbit secondary antibody (1:200; Jackson Immunoresearch Laboratories). Differences in innervation were determined by measuring area covered by Cy3-positive profiles $\left(\right.$ pixel $\left.^{2}\right)$ in 5 independent fields $(\times 20)$ from each genotype $(n=3)$ using Metamorph Premier software (Molecular Devices).

Enteric neuron studies in vitro. Enteric neural crest precursors were harvested from E14.5 Sprague Dawley rat (Charles River Laboratories) intestines using p $75^{\text {NTR }}$ antibody (53). Briefly, single-cell suspension was obtained after collagenase $(1 \mathrm{mg} / \mathrm{ml})$ and dispase $(1 \mathrm{mg} / \mathrm{ml})$ dissociation, followed by $\mathrm{p} 75^{\mathrm{NTR}}$ antibody incubation $\left(1: 1000,1\right.$ hour, $4^{\circ} \mathrm{C}$; gift from Moses Chao, New York University, New York, NY, USA) in B27-supplemented (Invitrogen) Neurobasal medium, and goat anti-rabbit coupled paramag- netic beads $\left(1: 50,1\right.$ hour, $4^{\circ} \mathrm{C}$; Miltenyi Biotec) for selective isolation. Immunoselected crest-derived cells were plated at a density of 1000 cells per well on poly-D-lysine/laminin-coated 8-well chamber slides (Biocoat; Fisher) in B27-supplemented Neurobasal medium plus GDNF $(50 \mathrm{ng} / \mathrm{ml})$ (54). We typically obtained $89 \%$ purity (P75-positive) using this method, and after 24 hours $80 \%$ of cells in culture were Ret-positive, consistent with previous studies $(55,56)$.

Lentivirus-mediated siRNA production and infections were as previously described (33). Briefly, cells were fixed 7 days after plating (6 days after siRNA treatment), and immunohistochemistry was performed with TuJ1 (1:1000; Covance) or Tau1 monoclonal antibody (1:100; Chemicon) to visualize neurons and axons using epifluorescence microscopy. Characterization of all the adaptor and scrambled control siRNAs including specificity and extent of knockdown has been previously described (33). The extent of knockdown of each of the adaptor RNAs was reconfirmed in cultured enteric neurons using quantitative RT-PCR in 2 to 3 independent experiments; Shc (95\% knockdown), Plcy (89\% knockdown), and Grb2 (90\% knockdown). Changes in neuron numbers were determined from a mean of 3 separate wells from 3 independent experiments. Statistical significance was determined using Student's $t$ test.

Microscopic and quantitative image analysis. Images for brightfield and immunofluorescence on tissue sections (Nikon Eclipse 80i) or wholemount specimens (Nikon SMZ1500) were captured (CoolSNAP ES camera for fluorescence and CoolSNAP CF for color images) and processed with Metamorph and Adobe Photoshop CS software using global adjustments in levels, contrast, or brightness.

Statistics. Statistical significance was determined with 2-tailed Student's $t$ test unless otherwise specified. For all studies, the sample size was 3 or more for each genotype. The values are represented as mean \pm SEM or as mean $\pm \mathrm{SD}$ as indicated, and $P<0.05$ was considered significant.

\section{Acknowledgments}

We thank Palvinder Kaur, Angela Lluka, and Nina Panchenko for excellent technical assistance. We are grateful to Hideki Enomoto for several discussions. This work was supported by an NIH George M. O'Brien Center for Kidney Disease Research grant (P30-DK079333) to Washington University, Children Discovery Institute grant MDII2009177 (to S. Jain), and NIH grants HD047396, DK081644 and DK082531 (to S. Jain), AG013730 (to J. Milbrandt), and DK57038, DK6459201 (to R.O. Heuckeroth).

Received for publication October 30, 2009, and accepted in revised form January 6, 2010.

Address correspondence to: Sanjay Jain, Washington University School of Medicine, Box 8126, 660 S. Euclid Ave., St. Louis, MO 63110. Phone: 314.454.8728; Fax: 314.454.7735; E-mail: sjain22@wustl.edu.
1. Madhani HD. Accounting for specificity in receptor tyrosine kinase signaling. Cell. 2001;106(1):9-11.

2. Ponder BA, Smith D. The MEN II syndromes and the role of the ret proto-oncogene. Adv Cancer Res. 1996;70:179-222.

3. Amiel J, et al. Hirschsprung disease, associated syndromes and genetics: a review. J Med Genet. 2008; 45(1):1-14.

4. Skinner MA, Safford SD, Reeves JG, Jackson ME, Freemerman AJ. Renal aplasia in humans is associated with RET mutations. Am J Hum Genet. 2008; 82(2):344-351.

5. Amiel J, Lyonnet S. Hirschsprung disease, associated syndromes, and genetics: a review. J Med Genet. 2001;
38(11):729-739.

6. Moore SW. The contribution of associated congenital anomalies in understanding Hirschsprung's disease. Pediatr Surg Int. 2006;22(4):305-315.

7. Prato AP, et al. Hirschsprung disease and congenital anomalies of the kidney and urinary tract (CAKUT): a novel syndromic association. Medicine. 2009; 88(2):83-90.

8. Baloh RH, Enomoto H, Johnson EM Jr, Milbrandt J. The GDNF family ligands and receptors - implications for neural development. Curr Opin Neurobiol. 2000;10(1):103-110.

9. Enomoto H, Heuckeroth RO, Golden JP, Johnson EM, Milbrandt J. Development of cranial parasympathetic ganglia requires sequential actions of GDNF and neurturin. Development. 2000;127(22):4877-4889.

10. Enomoto $\mathrm{H}$, et al. RET signaling is essential for migration, axonal growth and axon guidance of developing sympathetic neurons. Development. 2001; 128(20):3963-3974.

11. Jain $\mathrm{S}$, et al. Mice expressing a dominant-negative Ret mutation phenocopy human Hirschsprung disease and delineate a direct role of Ret in spermatogenesis. Development. 2004;131(21):5503-5513.

12. Sariola H, Saarma M. Novel functions and signalling pathways for GDNF. J Cell Sci. 2003;116(Pt 19):3855-3862.

13. Naughton CK, Jain S, Strickland AM, Gupta A, Mil- 
brandt J. Glial cell-line derived neurotrophic factormediated RET signaling regulates spermatogonial stem cell fate. Biol Reprod. 2006;74(2):314-321.

14. Cacalano G, et al. GFRalpha1 is an essential receptor component for GDNF in the developing nervous system and kidney. Neuron. 1998;21(1):53-62.

15. Enomoto $\mathrm{H}$, et al. GFR $\alpha 1$-deficient mice have deficits in the enteric nervous system and kidneys. Neuron. 1998;21(2):317-324

16. Moore MW, et al. Renal and neuronal abnormalities in mice lacking GDNF. Nature. 1996;382(6586):76-79.

17. Pichel JG, et al. Defects in enteric innervation and kidney development in mice lacking GDNF. Nature. 1996;382(6586):73-76

18. Sanchez MP, Silos-Santiago I, Frisen J, He B, Lira SA, Barbacid M. Renal agenesis and the absence of enteric neurons in mice lacking GDNF. Nature. 1996;382(6586):70-73.

19. Schuchardt A, D’Agati V, Larsson-Blomberg L, Costantini F, Pachnis V. Defects in the kidney and enteric nervous system of mice lacking the tyrosine kinase receptor Ret. Nature. 1994;367(6461):380-383.

20. Schuchardt A, D'Agati V, Pachnis V, Costantini F. Renal agenesis and hypodysplasia in ret-k- mutant mice result from defects in ureteric bud development. Development. 1996;122(6):1919-1929.

21. Jain S. The many faces of RET dysfunction in kidney. Organogenesis. 2009;5:1-14.

22. Airaksinen MS, Saarma M. The GDNF family: signalling, biological functions and therapeutic value. Nat Rev Neurosci. 2002;3(5):383-394.

23. Takahashi $M$. The GDNF/RET signaling pathway and human diseases. Cytokine Growth Factor Rev. 2001; 12(4):361-373.

24. Borrello MG, et al. Differential interaction of Enigma protein with the two RET isoforms. Biochem Biophys Res Commun. 2002;296(3):515-522.

25. Liu X, et al. Oncogenic RET receptors display different autophosphorylation sites and substrate binding specificities. J Biol Chem. 1996;271(10):5309-5312.

26. Rossel M, et al. Distinct biological properties of two RET isoforms activated by MEN 2A and MEN 2B mutations. Oncogene. 1997;14(3):265-275.

27. Tsui-Pierchala BA, Ahrens RC, Crowder RJ, Milbrandt J, Johnson EM Jr. The long and short isoforms of Ret function as independent signaling complexes. J Biol Chem. 2002;277(37):34618-34625.

28. de Graaff E, et al. Differential activities of the RET tyrosine kinase receptor isoforms during mammalian embryogenesis. Genes Dev. 2001;15(18):2433-2444.

29. Jain S, Encinas M, Johnson EM Jr, Milbrandt J. Critical and distinct roles for key RET tyrosine docking sites in renal development. Genes Dev. 2006;20(3):321-333.

30. Homma S, et al. Differential expression of the GDNF family receptors RET and GFRalpha1, 2, and 4 in subsets of motoneurons: a relationship between motoneuron birthdate and receptor expression. J Comp Neurol. 2003;456(3):245-259.

31. Wong A, et al. Phosphotyrosine 1062 is critical for the in vivo activity of the Ret9 receptor tyrosine kinase isoform. Mol Cell Biol. 2005;25(21):9661-9673.

32. Jijiwa $M$, et al. A targeting mutation of tyrosine 1062 in Ret causes a marked decrease of enteric neurons and renal hypoplasia. Mol Cell Biol. 2004; 24(18):8026-8036.

33. Gustin JA, et al. Deciphering adaptor specificity in GFL-dependent RET-mediated proliferation and neurite outgrowth. J Neurochem. 2007;102(4):1184-1194.

34. Ponder BA. The phenotypes associated with ret mutations in the multiple endocrine neoplasia type $2 \mathrm{syn}$ drome. Cancer Res. 1999;59(7 Suppl):1736s-1741s; discussion 1742 s.

35. Degl'Innocenti D, et al. Differential requirement of Tyr 1062 multidocking site by RET isoforms to promote neural cell scattering and epithelial cell branching. Oncogene. 2004;23(44):7297-7309.

36. Schuetz G, et al. The neuronal scaffold protein Shank3 mediates signaling and biological function of the receptor tyrosine kinase Ret in epithelial cells. J Cell Biol. 2004;167(5):945-952.

37. Besset V, Scott RP, Ibanez CF. Signaling complexes and protein-protein interactions involved in the activation of the Ras and phosphatidylinositol 3-kinase pathways by the c-Ret T receptor tyrosine kinase. J Biol Chem. 2000;275(50):39159-39166.

38. Lorenzo MJ, et al. RET alternate splicing influences the interaction of activated RET with the $\mathrm{SH} 2$ and PTB domains of Shc, and the SH2 domain of Grb2. Oncogene. 1997;14(7):763-771.

39. Encinas M, et al. Analysis of Ret knockin mice reveals a critical role for IKKs, but not PI $3-K$, in neurotrophic factor-induced survival of sympathetic neurons. Cell Death Differ. 2008;15(9):1510-1521.

40. Attie T, et al. Diversity of RET proto-oncogene mutations in familial and sporadic Hirschsprung disease. Hum Mol Genet. 1995;4(8):1381-1386.

41. Heanue TA, Pachnis V. Enteric nervous system development and Hirschsprung's disease: advances in genetic and stem cell studies. Nat Rev Neurosci. 2007; $8(6): 466-479$

42. Edery P, et al. Mutations of the RET proto-oncogene in Hirschsprung's disease. Nature. 1994 367(6461):378-380.

43. Geneste $\mathrm{O}$, et al. Two distinct mutations of the RET receptor causing Hirschsprung's disease impair the binding of signalling effectors to a multifunctional docking site. Hum Mol Genet. 1999;8(11):1989-1999.

44. Iwashita $T$, et al. Functional analysis of RET with hirschsprung mutations affecting its kinase domain. Gastroenterology. 2001;121(1):24-33.

45. Romeo $G$, et al. Point mutations affecting the tyrosine kinase domain of the RET proto-oncogene in Hirschsprung's disease. Nature. 1994
367(6461):377-378

46. McCallion AS, Stames E, Conlon RA, Chakravarti A. Phenotype variation in two-locus mouse models of Hirschsprung disease: Tissue-specific interaction between Ret and Ednrb. Proc Natl Acad Sci US A. 2003; 100(4):1826-1831

47. Basson MA, et al. Sprouty1 is a critical regulator of GDNF/RET-mediated kidney induction. Dev Cell. 2005;8(2):229-239.

48. Rozen EJ, Schmidt H, Dolcet X, Basson MA, Jain S, Encinas M. Loss of Sprouty1 rescues renal agenesis caused by Ret mutation. I Am Soc Nephrol. 2009; 20(2):255-259.

49. Orian-Rousseau V, Chen L, Sleeman JP, Herrlich $\mathrm{P}$, Ponta $\mathrm{H}$. CD44 is required for two consecutive steps in HGF/c-Met signaling. Genes Dev. 2002; 16(23):3074-3086.

50. Pandini G, Frasca F, Mineo R, Sciacca L, Vigneri $\mathrm{R}$, Belfiore A. Insulin/insulin-like growth factor I hybrid receptors have different biological characteristics depending on the insulin receptor isoform involved. J Biol Chem. 2002;277(42):39684-39695.

51. Vohra BP, Planer W, Armon J, Fu M, Jain S, Heuckeroth RO. Reduced endothelin converting enzyme-1 and endothelin-3 mRNA in the developing bowel of male mice may increase expressivity and penetrance of Hirschsprung disease-like distal intestinal aganglionosis. Dev Dyn. 2007;236(1):106-117.

52. Zhang B, et al. Mice lacking sister chromatid cohesion protein PDS5B exhibit developmental abnormalities reminiscent of Cornelia de Lange syndrome. Development. 2007;134(17):3191-3201.

53. Wu J, Chen J, Rothman T, Gershon M. Inhibition of in vitro enteric neuronal development by endothelin-3: mediation by endothelin B receptors. Development. 1999;126(6):1161-1173.

54. Vohra BPS, Fu M, Heuckeroth RO. Protein kinase $\mathrm{C}\{$ zeta $\}$ and glycogen synthase kinase- $3\{$ beta $\}$ control neuronal polarity in developing rodent enteric neurons, whereas SMAD specific E3 ubiquitin protein ligase 1 promotes neurite growth but does not influence polarity. J Neurosci. 2007;27(35):9458-9468.

55. Anitha M, et al. GDNF rescues hyperglycemiainduced diabetic enteric neuropathy through activation of the PI3K/Akt pathway. J Clin Invest. 2006; 116(2):344-356

56. Chalazonitis A, Rothman TP, Chen J, Gershon MD. Age-dependent differences in the effects of GDNF and NT-3 on the development of neurons and glia from neural crest-derived precursors immunoselected from the fetal rat gut: expression of GFRalpha-1 in vitro and in vivo. Dev Biol. 1998; 204(2):385-406.

57. Jain S, et al. Mice expressing a dominant-negative Ret mutation phenocopy human Hirschsprung disease and delineate a direct role of Ret in spermatogenesis. Development. 2004;131(21):5503-5513. 